\title{
ASSESSMENT OF VOLTAGE QUALITY INDICATORS IN UNBALANCED AND HARMONIC DISTORTED REGIME
}

\author{
${ }^{1,2}$ Sorin ABAGIU, ${ }^{1}$ Ionel LEPADAT, ${ }^{2}$ Elena HELEREA, ${ }^{1}$ Cătălin MIHAI \\ ${ }^{1}$ Electricity Distribution Subsidiary of Electrica Distribution Transilvania South SA, Brasov \\ Romania, e-mail: sabagiu41@gmail.com, ionellepadat@gmail.com, catalin77@gmail.com \\ ${ }^{2}$ Department of Electrical Engineering and Applied Physics, Faculty of Electrical Engineering \\ and Computer Science, Transilvania University of Brasov, 29, B-dul Eroilor, 500036, Brasov \\ Romania, e-mail: helerea@unitbv.ro
}

Received 6 March 2015; accepted 22 May 2015

\begin{abstract}
This paper deals with the development of a model for assessment of the indicators, which characterize the unbalanced and harmonic distorted operation regime in the three-phase low voltage networks. The symmetrical components transformation and the Fourier method are applied, in which each harmonic is decomposed into zero, positive and negative sequence components. The quality indicators in voltage distorted and unbalanced regime are obtained using MathCAD software. The proposed model and procedure is applied for specific case studies. This approach allows the identification and analysis of distorted and unbalanced regime indicators of three-phase low voltage supply system in common coupling point of the consumer, and the procedure can be integrated into a power quality monitoring system.
\end{abstract}

Keywords: Voltage quality indicators, Unbalanced and harmonic distorted regime, Low three-phase voltage network, Symmetrical component method, Fourier decomposition method

\section{Introduction}

Currently, the three-phase low voltage networks, which supply residential, commercial and industrial consumers are characterized by the presence of voltage and current harmonics, often accompanied by unbalances. This is due to the introduction of widespread power electronic devices, the presence of a large number of single-phase consumers, and the development of distributed power systems, in which the electrical consumers are active elements.

Effects in a power network operating in unbalanced regime are similar to the effects of distorted regimes, namely, to increase power and energy losses, with negative 
consequences for the efficiency of transfer of power. The unbalanced and distorted regime of the power supply to consumer affects operating parameters of consumer equipment, which are usually designed to operate under sinusoidal and balanced regime.

To mitigate the effects of distorted and unbalanced regime were conducted research on developing methods and tools to increase the power quality delivered at the common coupling point [1]-[3].

Study of voltage unbalance allowed standardization of unbalance indicators and assessment methods in case of low voltage power network [4]-[10]. Also, the modeling of harmonic currents, the analysis of their effects and introducing new sets of indicators able to characterize power quality in terms of harmonic distortion are the subject of many researches [11]-[14].

But a pertinent analysis of power quality indicators can only be done if the unbalance is analyzed together with distorted regime. There are papers [15]-[17], analyzed unbalanced and distorted regime and established the impact of non-linear loads on the power distribution network and substantiation. Also, in international regulations [18]-[19], are defined specific indicators, and assessing the possible errors in the calculation of indicators are taken in account. The researches in papers [20]-[23] target on the development of hardware and software algorithms to determine the RMS values of the voltages and currents in combined regime of unbalance and harmonic distorted regime, and identify possible hidden errors in calculation and interpretation.

All these studies indicate that distorted and unbalanced regimes are complex processes that require a detailed characterization.

The aim of this paper is to develop a tool for assessment of the voltage quality indicators, which characterize the distorted and unbalanced regime of operation of the three-phase low voltage networks. Quality indicators are established in MathCAD applying the symmetrical components transformation and the Fourier method, in which each harmonic is decomposed into zero, positive and negative sequence components.

\section{Voltage quality indicators}

The unbalanced and non-sinusoidal regime of the phase voltages of a three-phase power system is described by considering the Fourier series decomposition, with relationships:

$$
\begin{aligned}
& u_{R}(t)=\sqrt{2} \cdot \sum_{k=1}^{\infty} U_{R}^{k} \sin \left(k \omega t+\varphi_{R}^{k}\right), \\
& u_{S}(t)=\sqrt{2} \cdot \sum_{k=1}^{\infty} U_{S}^{k} \sin \left(k\left(\omega t-\frac{2 \pi}{3}\right)+\varphi_{S}^{k}\right), \\
& u_{T}(t)=\sqrt{2} \cdot \sum_{k=1}^{\infty} U_{T}^{k} \sin \left(k\left(\omega t+\frac{2 \pi}{3}\right)+\varphi_{T}^{k}\right),
\end{aligned}
$$


where $k$ is the number of the harmonic, $U_{R}^{k}$ is the corresponding RMS phase voltage harmonic of order $k$ and $\varphi^{k}$ is the initial phase angle of the harmonic voltage of order $k$. The reference angle for the entire system is considered as the origin of phase voltage component at the fundamental frequency [22]. For three-phase voltage systems there is a correlation (Table I) between the harmonic order and sequence of rotation $\omega$ [17].

\section{Table I}

Correlation between the harmonic order and the rotation sequence

\begin{tabular}{|c|c|l|}
\hline $\begin{array}{c}\text { Order of } \\
\text { harmonics }\end{array}$ & $\begin{array}{c}\text { Frequency of } \\
\text { rotation }\end{array}$ & $\begin{array}{c}\text { Sequence of } \\
\text { rotation }\end{array}$ \\
\hline $3 n+1$ & $(3 n+1) \omega$ & Positive \\
$3 n+2$ & $(3 n+2) \omega$ & Negative \\
$3 n+3$ & $(3 n+3) \omega$ & Zero \\
\hline
\end{tabular}

The specific indicators that characterize unbalanced and distorted regime is determined by applying the method using symmetrical components and harmonics properties described in Table I.

Considering the relation (1), the matrix of phase voltage phasors corresponding to $k$ harmonic order is defined:

$$
\mathbf{U}^{k}=\left[\begin{array}{lll}
\mathbf{U}_{R}^{k} & \mathbf{U}_{S}^{k} & \mathbf{U}_{T}^{k}
\end{array}\right]^{t}
$$

where $t$ indicates matrix transposition.

To separate the balanced and unbalanced system components, the following considerations are used.

\subsection{Distorted and balanced voltage regime}

In balanced regime, the equilibrium conditions of the RMS values and phase angles for phase voltages are:

$$
U_{R}^{k}=U_{S}^{k}=U_{T}^{k}, \quad \text { and } \quad \varphi_{R}^{k}=\varphi_{S}^{k}=\varphi_{T}^{k},
$$

where for $=0,1, \cdots, \infty$, the following properties are valid:

- Harmonics of order $k=3 \mathrm{n}+1, \mathrm{n}=0,1,2, \ldots$, of the voltage phasors rotate positively with the frequency $f$ of the network. Positive sequence components contained in these harmonics will be non-zero and negative and zero sequence components will be zero;

- Harmonics of order $k=3 n+2, n=0,1,2, \ldots$, of the voltage and current phasors rotate negatively with the frequency $f$ of the network. Negative sequence 
component contained in these harmonics will be non-zero and positive and zero sequence components will be zero;

- Harmonics of order $k=3 \mathrm{n}+3, \mathrm{n}=0,1,2, \ldots$, the voltage and current phasors not rotate, are in phase. Zero sequence component contained in these harmonics will be non-zero and positive and negative sequence components will be zero.

The balanced regime can be characterized by:

- $\quad$ positive sequence components corresponding harmonic order $k=3 n+1$;

- $\quad$ negative sequence components corresponding harmonic order $k=3 n+2$;

- zero sequence components corresponding harmonic order $k=3 n+3$.

To calculate the symmetrical components for each group of harmonics are defined the transformation matrices:

$-\quad$ for $k=3 n+1$

$$
\mathbf{T}_{1}=\frac{1}{3}\left[\begin{array}{ccc}
1 & a & a^{2} \\
1 & a^{2} & 1 \\
1 & 1 & 1
\end{array}\right]
$$

$-\quad$ for $k=3 n+2$

$$
\begin{aligned}
\mathbf{T}_{2} & =\frac{1}{3}\left[\begin{array}{ccc}
1 & a^{2} & a \\
1 & a & a^{2} \\
1 & 1 & 1
\end{array}\right] ; \\
& -\quad \text { for } k=3 \mathrm{n}+3
\end{aligned}
$$

$$
\mathbf{T}_{0}=\frac{1}{3}\left[\begin{array}{ccc}
1 & 1 & 1 \\
1 & \frac{-1-\sqrt{3}}{2} & \frac{-1+\sqrt{3}}{2} \\
1 & \frac{-1+\sqrt{3}}{2} & \frac{-1-\sqrt{3}}{2}
\end{array}\right],
$$

where $a=e^{j \frac{2 \pi}{3}}$.

The relations (4)-(6) are applied for each group of harmonic components, and the following symmetrical voltage components are obtained: 
- phase voltage $(k=3 n+1)$ components, for positive sequence harmonics:

$\left[\begin{array}{lll}U_{p 1}^{3 n+1} & U_{p 1}^{3 n+2} & U_{p 1}^{3 n+3}\end{array}\right]^{t}=\mathbf{T}_{1}\left[\begin{array}{lll}\mathbf{U}_{R}^{3 n+1} & \mathbf{U}_{S}^{3 n+1} & \mathbf{U}_{T}^{3 n+1}\end{array}\right]^{t}$,

- phase voltage $(k=3 n+2)$ components, for negative sequence harmonics:

$\left[\begin{array}{lll}U_{p 2}^{3 n+1} & U_{p 2}^{3 n+2} & U_{p 2}^{3 n+3}\end{array}\right]^{t}=\mathbf{T}_{2}\left[\begin{array}{lll}\mathbf{U}_{R}^{3 n+2} & \mathbf{U}_{S}^{3 n+2} & \mathbf{U}_{T}^{3 n+2}\end{array}\right]^{t}$,

- phase voltage $(k=3 n+3)$ components, for zero sequence harmonics:

$\left[\begin{array}{lll}U_{p 0}^{3 n+1} & U_{p 0}^{3 n+2} & U_{p 0}^{3 n+3}\end{array}\right]^{t}=\mathbf{T}_{0}\left[\begin{array}{lll}\mathbf{U}_{R}^{3 n+3} & \mathbf{U}_{S}^{3 n+3} & \mathbf{U}_{T}^{3 n+3}\end{array}\right]^{t}$

With these considerations, the following phase voltage characteristics corresponding to the distorted and balanced regime are defined.

- Phase voltage equivalent to balanced regime:

$U_{p}^{b}=\sqrt{\sum_{n=0}^{\infty}\left[\left(U_{p 1}^{3 n+1}\right)^{2}+\left(U_{p 2}^{3 n+2}\right)^{2}+\left(U_{p 0}^{3 n+3}\right)^{2}\right]}$,

where

- the first term corresponds to a positive component of harmonics with positive sequence rotation;

- the second term corresponds to the negative component of harmonics with negative sequence harmonic rotation;

- the third term corresponds to the zero component of harmonics with zero sequence harmonics rotation;

- Phase voltage equivalent to balanced regime for fundamental harmonic:

$U_{p}^{b 1}=U_{p 1}^{1}$

- Distorted residue of phase voltage equivalent to balanced regime:

$(\delta U)_{p}^{b d}=\sqrt{\left(U_{p}^{b}\right)^{2}-\left(U_{p}^{b 1}\right)^{2}}$ 


\subsection{Distorted and unbalance voltage regime}

In the distorted regime in the presence of unbalance no longer a connection between harmonic order and direction of rotation of the corresponding phasors exists. Even for fundamental harmonic in unbalanced regime there are three components of positive, negative and zero sequence. It must be considered appropriate components of positive, negative and zero sequence.

In voltage distorted and unbalanced regime the following phase voltage quality indicators are defined:

- Phase voltage equivalent to unbalanced regime:

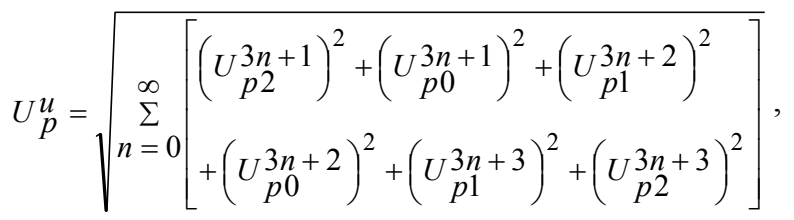

where the terms correspond to all harmonic components, which do not affect the balanced regime.

- Phase voltage equivalent to unbalanced regime for fundamental harmonic:

$$
U_{p}^{u 1}=\sqrt{\left(U_{p 2}^{1}\right)^{2}+\left(U_{p 0}^{1}\right)^{2}} ;
$$

- Deformed residue of phase voltage equivalent to unbalanced regime:

$$
(\delta U)_{p}^{u d}=\sqrt{\left(U u_{p}^{u}\right)^{2}-(U \underset{p}{u 1})^{2}}
$$

- Total phase voltage equivalent to unbalanced and distorted regime:

$$
U_{p}^{u d}=\sqrt{\left[(\delta U)_{p}^{b d}\right]^{2}+\left[(\delta U)_{p}^{u d}\right]^{2}} .
$$

The voltage distortion and unbalance factors are also defined:

- Distortion factor of phase voltage equivalent to unbalanced regime:

$$
k_{U}^{u}=\frac{U_{p}^{u}}{U_{p}^{u 1}}
$$

- Unbalance indicator of phase voltage corresponding to fundamental harmonic: 
$k_{U}^{u d 1}=\frac{U_{p}^{u 1}}{U_{p}^{b 1}} ;$

- Total Harmonic Distortion indicator of phase voltage equivalent to balanced regime:

$\left(T H D_{U}\right)^{b}=\frac{(\delta U)_{p}^{b d}}{U_{p}^{b 1}}$

- Total Harmonic Distortion indicator of phase voltage equivalent to unbalanced regime:

$\left(T H D_{U}\right)^{\mu}=\frac{(\delta U)_{p}^{u d}}{U_{p}^{u 1}}$

- Total indicator of phase voltage equivalent to unbalanced regime:

$\left(T P U_{U}\right)^{u}=\frac{U_{p}^{u}}{U_{p}^{b}}$

- Total indicator phase voltage equivalent to distorted regime:

$$
\left(\operatorname{TPD}_{U}\right)^{u}=\frac{U_{p}^{u d}}{\sqrt{\left(U_{p}^{b 1}\right)^{2}+\left(U_{p}^{u 1}\right)^{2}}} .
$$

The system of relationships (10)-(22) shows that the analysis of three-phase voltage system in unbalanced and distorted regime is complex and requires laborious calculations, with specific and dedicated software. In this paper an application in MathCAD environment is developed to obtain the voltage characteristics and indicators.

\section{Computation tool}

The flowchart of the calculation modulus of the characteristics and indicators of in phase voltage in an unbalanced and distorted regime is shown in Fig. 1. 


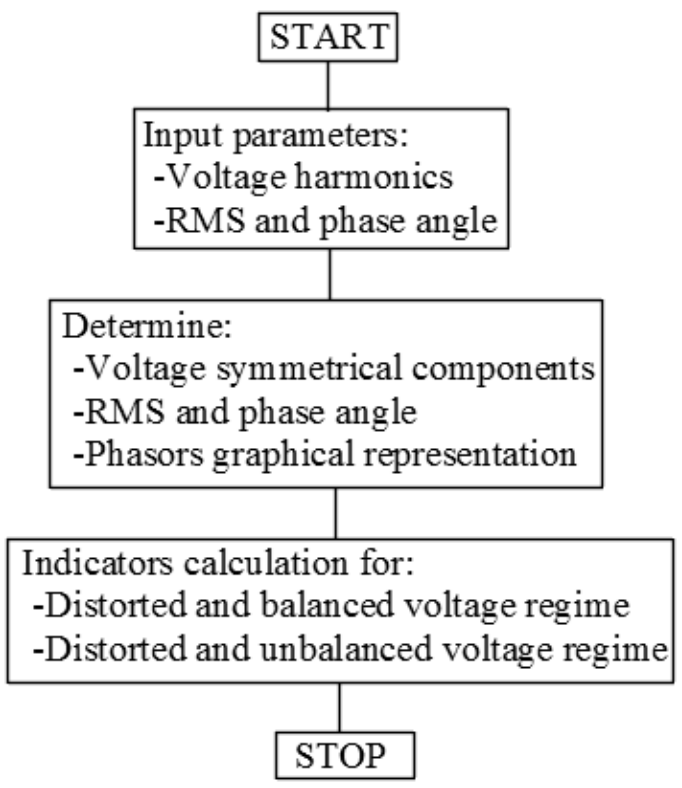

Fig. 1. Flow chart for voltage indicators calculation in distorted and unbalanced regime

The input data are RMS and phase angles values of voltage harmonics $\left(U_{R}^{k}, U_{S}^{k}, U_{T}^{k}, \varphi_{R}^{k}, \varphi_{S}^{k}, \varphi_{T}^{k}\right)$. Applying transformation matrix (4)-(6), with the relations (4)-(9), the symmetrical voltage components $U_{1}^{k}, U_{2}^{k}, U_{0}^{k}$ for each voltage harmonic are obtained.

The phase voltage quality indicators are determined with the relations (10)-(16), and the voltage distortion and unbalance factors are obtained with the relations (17)-(22).

\section{Case studies}

To illustrate the calculation method of power quality indicators, four case studies of the operation regimes in a low three-phase voltage network are considered:

- Case 1 - balanced regime without harmonic distortions;

- Case 2 - unbalanced regime without harmonic distortions;

- Case 3 - balanced regime with harmonic distortions (harmonics 3 and 5);

- Case 4 - unbalanced regime with harmonic distortions (harmonics 3 and 5).

Only voltage unbalance defined by inequality of RMS phase's voltage is considered. In Table II the RMS phase voltages and corresponding phase angles values for the four case studies are given.

The waveforms of three-phase voltage systems corresponding to the four considered regimes are shown in Fig. 2. 
Table II

Input data for the four case studies

\begin{tabular}{|c|c|c|c|c|c|c|c|c|}
\hline Voltage & Case 1 & Case 2 & \multicolumn{3}{|c|}{ Case 3 } & \multicolumn{3}{c|}{ Case 4 } \\
\hline Harmonics & 1 -st & 1-st & 1-st & 3 rd & 5 -th & 1-st & 3-rd & 5 -th \\
\hline$U_{R}^{k}[\mathrm{~V}]$ & 230 & 210 & $\begin{array}{c}230 \\
0^{\circ}\end{array}$ & $\begin{array}{c}46.0 \\
0^{\circ}\end{array}$ & $\begin{array}{c}23.0 \\
0^{\circ}\end{array}$ & $\begin{array}{c}210 \\
0^{\circ}\end{array}$ & $\begin{array}{c}57.5 \\
0^{\circ}\end{array}$ & $\begin{array}{c}34.5 \\
0^{\circ}\end{array}$ \\
$\varphi_{R}^{k}$ & $0^{\circ}$ & $0^{\circ}$ & 230 & 46.0 & 23.0 & 228 & 46.0 & 23.0 \\
\hline$U_{S}^{k}[\mathrm{~V}]$ & 230 & 228 & 230 & $-120^{\circ}$ & $-120^{\circ}$ \\
$\varphi_{S}^{k}$ & $-120^{\circ}$ & $-120^{\circ}$ & $-120^{\circ}$ & $-120^{\circ}$ & $-120^{\circ}$ & $-120^{\circ}$ & $-120^{\circ}$ & \\
\hline$U_{T}^{k}[\mathrm{~V}]$ & 230 & 240 & 230 & 46.0 & 23.0 & 240 & 34.5 & 11.5 \\
$\varphi_{T}^{k}$ & $120^{\circ}$ & $120^{\circ}$ & $120^{\circ}$ & $120^{\circ}$ & $120^{\circ}$ & $120^{\circ}$ & $120^{\circ}$ & $120^{\circ}$ \\
\hline
\end{tabular}

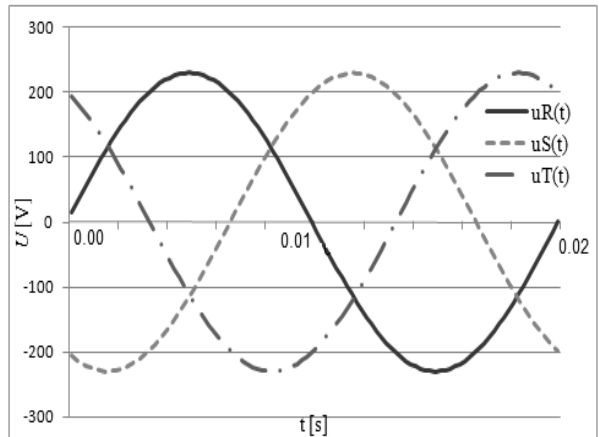

Case 1

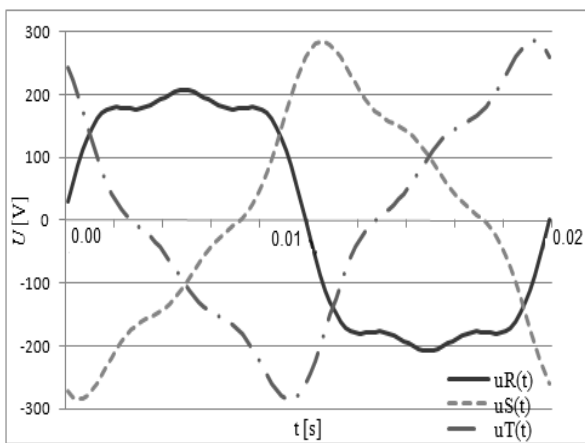

Case 3

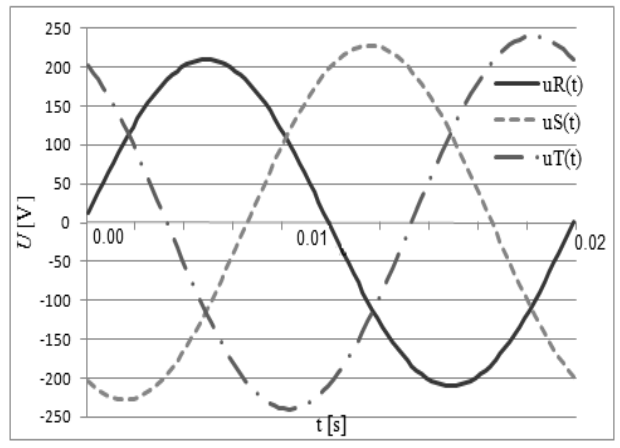

Case 2

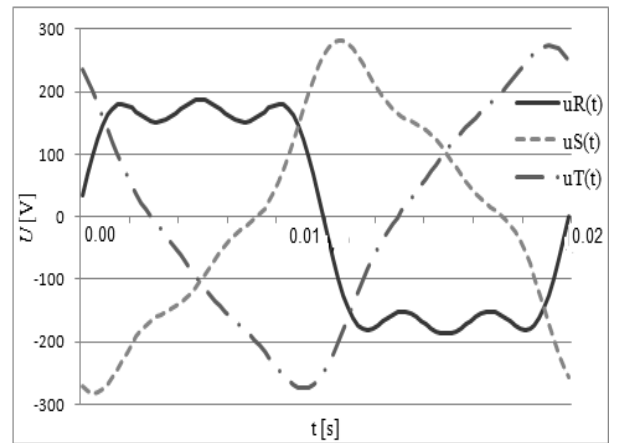

Case 4

Fig. 2. Waveforms of three-phase voltage systems in the four case studies 
Applying the algorithm described in Paragraph 2, the voltage quality indicators are calculated. The results are shown in Table III and Table IV.

Table III

The phase voltage quality indicators for the four case studies

\begin{tabular}{|c|c|c|c|c|}
\hline & Case 1 & Case 2 & Case 3 & Case 4 \\
\hline$U_{p}^{b}$ & 230 & 226 & 232,29 & 228,04 \\
$U_{p}^{b 1}$ & 230 & 226 & 230 & 226.00 \\
$(\delta U)_{p}^{b d}$ & 0 & 0 & 32.53 & 30.45 \\
$U_{p}^{u}$ & 0 & 12.33 & 32.53 & 37.80 \\
$U_{p}^{u 1}$ & 0 & 0 & 0 & 6.64 \\
$(\delta U)_{p}^{u d}$ & 0 & 12.33 & 32.33 & 37.21 \\
$U \underset{p}{u d}$ & 0 & 12.33 & 46 & 48.08 \\
\hline
\end{tabular}

Table IV

The voltage distortion and unbalance factors for the four case studies

\begin{tabular}{|c|c|c|c|c|}
\hline & Case 1 & Case 2 & Case 3 & Case 4 \\
\hline$k_{U}^{u}$ & 0 & 0.055 & 0.14 & 16.73 \\
$k_{U}^{u d} 1$ & 0 & 0 & 0 & 2.94 \\
$\left(T H D_{U}\right)^{b}$ & 0 & 0 & 14.00 & 13.47 \\
$\left(T H D_{U}\right)^{u}$ & 0 & 0.055 & 14.00 & 16.46 \\
$\left(T P U_{U}\right)^{u}$ & 0 & 0.055 & 14.00 & 16.57 \\
$\left(T P D_{U}\right)^{u}$ & 0 & 0.055 & 20.00 & 21.27 \\
\hline
\end{tabular}

The results underline the following:

- Case 1 - Balanced regime without distortions

- equivalent phase voltage $\left(U \begin{array}{l}b \\ p\end{array}\right)$ and the equivalent phase voltage of fundamental $\left(U \begin{array}{c}b 1 \\ p\end{array}\right)$ are equal to the nominal value of supply phase voltage;

- other voltage quality indicators and distortion and unbalance factors are zero, indicating no distorted and unbalanced regime.

- Case 2 - Unbalanced regime without distortions

- distorted residue of phase voltage equivalent to balanced regime $(\delta U)_{p}^{b d}$ is zero, indicating the lack of distorted regime; 
- phase voltage equivalent to unbalanced regime $\left(U_{p}^{u}\right)$ indicates the voltage unbalance;

- phase voltage equivalent to unbalanced regime $U_{p}^{u}$, the distorted residue of equivalent phase voltage under unbalanced regime $(\delta U)_{p}^{u d}$ and total equivalent phase voltage under unbalanced and distorted regime $U \underset{p}{u d}$ have the same value, indicating that the voltage unbalance is given by the presence of unbalanced regime;

- Case 3 - Unbalanced regime with voltage harmonics

- distorted residue of phase voltage equivalent to balanced regime $(\delta U)_{p}^{b d}$ indicates the level of distorted regime;

- distorted residue of phase voltage equivalent to balanced regime $(\delta U)_{p}^{b d}$. phase voltage equivalent to unbalanced regime $U_{p}^{u}$ and the distorted residue of equivalent phase voltage under unbalanced regime $(\delta U)_{p}^{u d}$ are equal, indicating that unbalance is caused by the presence of distortions;

- phase voltage distortion factor equivalent to balanced regime $\left(T H D_{U}\right)^{b}$ is equal to the total harmonic distortion (THD) in balanced regime;

- total indicator of unbalanced regime of equivalent phase voltage $\left(T P U_{U}\right)^{u}$ and total indicator of distorted regime of equivalent phase voltage $\left(T P D_{U}\right)^{u}$ indicate that the predominant regime is distorted.

- Case 4 - Unbalanced and distorted regime with voltage harmonics

- total equivalent phase voltage under unbalanced and distorted regime $U_{p}^{u d}$ indicates that the prevailing regime is distorted regime;

- total indicator of unbalanced regime of equivalent phase voltage $\left(T P U_{U}\right)^{u}$ and total indicator of distorted regime of equivalent phase voltage $\left(T P D_{U}\right)^{u}$ indicate that the predominant is distorted regime.

\section{Conclusions}

The unbalanced regime with harmonic distortions in low three-phase voltage networks requires an adequate monitoring of power quality using a set of appropriate indicators.

In this paper the analysis on the phase voltage waveforms of three-phase low voltage network which operates in unbalanced and distorted regime is done using the 
symmetrical component method and Fourier decomposition. The characteristics and indicators of unbalance and harmonic distortion regime are obtained.

The set of determined indicators allows to characterize unbalanced and distorted regimes, with the following observations and comments:

- $\quad$ without harmonic distortions, the $k_{U}^{u d 1}$ indicator is equal to the ratio of negative sequence current to positive sequence, which can be a common indicator of unbalanced system;

- $\quad T P D_{U}$ and $T P U_{\mathrm{U}}$ indicators can provide information regarding the difference between three-phase systems unbalance caused by unbalances and harmonic distortion.

The results of this study may be useful in:

- characterization of unbalance and harmonic distortion regime in power systems;

- $\quad$ setting industry standards for the operation of sensitive loads in power systems;

- power quality study such as the design and construction of systems with balanced loads based on the proposed method.

\section{References}

[1] Golovanov C., Albu M. Modern issues on measurements in power systems (In Romanian), Bucureşti, Ed. Tehnică, 2001.

[2] Andrei M., Chicco G., Napoli R., Porumb R., Postolache P., Toader C. Technical and economic challenges regarding the regulation of electrical energy quality, Energetica, Vol. 52, No. 2, 2004, pp. 50-61.

[3] Baggini A. Handbook of power quality, John Wiley \& Sons Ltd, 2008.

[4] Pillay P., Manyage M. Definitions of voltage unbalance, IEEE Power Engineering Review, Vol. 5, 2001, pp. 50-51.

[5] Jouanne A., Banerjee B. Assessment of voltage unbalance, IEEE Trans. on Power Delivery, Vol. 16, No. 4, 2001, pp. 782-790.

[6] Pokorny M. Analysis of unbalance due to asymmetrical loads, Iranian Journal of Electrical and Computer Engineering, Vol. 4, No. 1, 2005, pp. 50-56.

[7] Gosbel V. J., Herath H. M. S. C., Perera B. S. P., Robinson D. A. Sources of error in unbalance measurements, Proc. Australasian Universities Power Engineering Conference (AUPEC), Producing Quality Electricity for Mankind, Australasian Committee for Power Engineering, Victoria, Australia, 2002, pp. 1-6, http://ro.uow.edu.au/infopapers/1200/ (last visited 12 February 2015).

[8] Olczykowski Z., Wasowski A. Methods of voltage unbalance estimation in electric power networks, Electrical Power Quality and Utilization Journal, Vol. XII, No. 2, 2006, pp. 71-76.

[9] Koch R., Beaulieu G., Berthet L., Halpin M. International survey of unbalance levels in LV, MV, HV, and EHV power systems, CIGRE/CIRED JWG C4.103 Results, In: 19th International Conference on Electricity Distribution, Vienna, Austria, 21-24 May 2007, pp. $1-4$.

[10] Garcia D. C., Filho A. L. F., Oliveira A. G. M., Fernandes O. A., Francisco A. Voltage unbalance numerical evaluation and minimization, Electric Power Systems Research, Vol. 79, 2009, pp. 1441-1445. 
[11] Liew A. C. Excessive neutral currents in three-phase fluorescent lighting circuits, IEEE Trans. on Ind. Appl, Vol. 25, No. 4, 1989, pp. 776-782.

[12] Carbone R., Menniti D., Morrison R. E., Delaney E. Harmonic and inter-harmonic distortion in current source type inverter drives, IEEE Transactions on Power Delivery, Vol.

No. 3, 1995, pp. 1576-1583.

[13] Istók R. Measurement systems for high frequency noise emission of compact fluorescent lamps, Pollack Periodica, Vol. 8, No. 2, 2013, pp. 173-178.

[14] Paula Silva (de) S. F., Oliveira (de) J. C. The sharing of responsibility between the supplier and the consumer for harmonic voltage distortion, A case study, Electric Power Systems Research, Vol. 78, 2008, pp. 1959-1964.

[15] Zheng T., Makram E. B., Girgis A. A. Evaluating power system unbalance in the presence of harmonic distortion, IEEE Trans. on Power Delivery, Vol. 18, No. 2, 2003, pp. 393-397.

[16] Dell' Aquila A., Marinelli M., Giuseppe V., Yanchetta P. New power-quality assessment criteria for supply systems under unbalanced and non-sinusoidal conditions, IEEE Trans. on Power Delivery, Vol. 19, No. 3, 2004, pp. 1284-1290.

[17] Chicco G., Postolache P., Toader C. Analysis of three-phase systems with neutral under distorted and unbalanced conditions in the symmetrical component-based framework, IEEE Trans. on Power Delivery, Vol. 22, No. 1, 2007, pp. 674-683.

[18] IEC 61000-4-30:2003, Electromagnetic compatibility (EMC), Part 4-30, Testing and measurement techniques, Power quality measurement methods.

[19] IEEE 1459:2000, IEEE Trial-use standard definitions for the measurement of electric power quantities under sinusoidal, non-sinusoidal, balanced, or unbalanced conditions.

[20] Albu M., Heydt G. T. On the use of RMS values in power quality assessment, IEEE Trans. on Power Delivery, Vol. 18, No. 4, 2003, pp. 1586-1587.

[21] Apetrei D., Chicco G., Neurohr R., Albu M., Postolache P. Power quality monitoring, Data relevance and usefulness, MELECON 2010 - The 15th IEEE Mediterranean Electrotechnical Conference, Valletta, Malta, 26-28 April 2010, pp. 1630-1635.

[22] Batrinu F., Chicco G., Ciortea A.O., Porumb R., Postolache P., Spertino F., Toader C. Experimental evaluation of unbalance and distortion indicators in three-phase systems with neutral, Power Tech. 2007, IEEE, Lausanne, Switzerland, 1-5 July 2007, pp. 1486-1491.

[23] Lepadat I.,Helerea E., Moasa B. Software application for unbalanced regime analysis in three phase low voltage networks, Pollack Periodica, Vol. 8, No. 2, 2013, pp. 149-161. 